\title{
Metabolic effects of mirabegron in mice: implications for use in diabetes
}

\author{
Dana S Hutchinson ${ }^{1}$, Nodi Dehvari ${ }^{2}$, Masaaki Sato ${ }^{1}$, Jie Gao ${ }^{1}$, Lynda Whiting ${ }^{1}$, Saori Mukaida ${ }^{1}$, \\ Jon Merlin ${ }^{1}$, Roger J Summers ${ }^{1}$, Bronwyn A Evans ${ }^{1}$, Tore Bengtsson ${ }^{2}$ \\ ${ }^{I}$ Drug Discovery Biology, Monash University, Australia, ${ }^{2}$ Department of Molecular Biosciences, The Wenner-Gren \\ Institute, Stockholm University, Stockholm, Sweden
}

Introduction: The beta3-adrenoceptor was initially a target for obesity and diabetes treatment, but several beta3adrenoceptor agonists failed in clinical trials due to their lack of efficacy at the human beta3-adrenoceptor. Recently, the beta3-adrenoceptor agonist mirabegron has been approved for use in humans for overactive bladder, but there is very limited knowledge of any effects mirabegron has on metabolic parameters in model systems.

Methods; We have investigated the actions of mirabegron in brown, white and brite adipocytes from mice, using cAMP assays, qPCR for Ucp1 mRNA content, glucose uptake using 3H-2-deoxyglucose, Seahorse xF96 analysis for in vitro oxygen consumption and glycolysis measurements. We have assessed whether the effects of mirabegron in vivo (glucose uptake, oxygen consumption, glucose tolerance tests) are due to activation of the beta3-adrenoceptor with the use of beta3-adrenoceptor knockout mice.

Results: Mirabegron increases cyclic AMP levels, Ucp1 mRNA content, glucose uptake and cellular glycolysis in brown adipocytes, and this effect is significantly absent/reduced in white adipocytes. In brite adipocytes, mirabegron increases cyclic AMP levels, Ucp1 mRNA content leading to increased UCP1 mediated oxygen consumption, glucose uptake and cellular glycolysis. Mirabegron in vivo increases whole body oxygen consumption rates, glucose uptake into brown and inguinal white adipose tissue, and improves glucose tolerance, which are dependent upon the presence of the beta3adrenoceptor.

Conclusion: Mirabegron has the potential to be used for further studies examining its effects in metabolic disease, specifically in humans. 